\title{
An Inverse Mixed Impedance Scattering Problem in a Chiral Medium
}

\author{
Evagelia S. Athanasiadou
}

check for

updates

Citation: Athanasiadou, E.S. An Inverse Mixed Impedance Scattering Problem in a Chiral Medium.

Mathematics 2021, 9, 104.

https://doi.org/10.3390/ math9010104

Received: 8 November 2020 Accepted: 31 December 2020 Published: 5 January 2021

Publisher's Note: MDPI stays neutral with regard to jurisdictional claims in published maps and institutional affiliations.

Copyright: (c) 2021 by the author. Licensee MDPI, Basel, Switzerland. This article is an open access article distributed under the terms and conditions of the Creative Commons Attribution (CC BY) license (https: / / creativecommons.org / licenses/by/4.0/).
Department of Mathematics, National and Kapodistrian University of Athens, 15784 Athens, Greece; eathan@math.uoa.gr

\begin{abstract}
An inverse scattering problem of time-harmonic chiral electromagnetic waves for a buried partially coated object was studied. The buried object was embedded in a piecewise isotropic homogeneous background chiral material. On the boundary of the scattering object, the total electromagnetic field satisfied perfect conductor and impedance boundary conditions. A modified linear sampling method, which originated from the chiral reciprocity gap functional, was employed for reconstruction of the shape of the buried object without requiring any a priori knowledge of the material properties of the scattering object. Furthermore, a characterization of the impedance of the object's surface was determined.
\end{abstract}

Keywords: inverse scattering; reciprocity gap functional; chiral media; mixed boundary conditions MSC: 35Q60; 35R30; 78A46; 35P25

\section{Introduction}

In this work, the inverse electromagnetic scattering problem of determining the surface impedance and the shape of a buried partially coated scattering object in chiral media is studied. In order to do this, we need information of the value of the electric and magnetic fields on the surface of the earth.

A chiral material is one that displays optical activity such that, when the plane of vibration of a linearly polarized light passes through an opticaly active medium is rotated. Over the last few years, chiral materials have been studied more intensely and there are more studies on the subject, covering both their applications and their theoretical background. Furthermore, various papers have been written on direct and inverse electromagnetic scattering problems for chiral media. Indicatively, we refer to Reference [1-4]. These materials are characterized by a set of two constitutive equations, in which electric and magnetic fields are connected via a physical variable or constant, known as chirality.

In this work, Drude-Born-Fedorov constitutive equations are used, as they are symmetric under time reversality and duality transformation [5]. In homogeneous and isotropic chiral media, the electric and magnetic fields are a combination of Left Circularly Polarized and Right Circularly Polarized components that have different phase speeds. So, in the applications, we can use the Bohren decomposition [6] of electric and magnetic fields into suitable Left and Right Circularly Beltrami fields. Such fields have been employed in Reference [2] for the definition of the chiral Herglotz wave functions and in Reference [6] for formulation of the electric dipole, which both play an important role in the present work. In Reference [7], the measure of chirality for a certain class of chiral scatterers has been calculated, while, in Reference [8], properties of chiral metamaterials are described. In addition, we note that Ammari and Nédélec in Reference [1] have proved that the wellknown Silver-Müller radiation condition remains valid in chiral media. In Reference [4,9], the direct and the inverse electromagnetic scattering problems by a mixed impedance screen in a chiral environment are investigated, respectively. Beltrami fields have been used for the uniqueness and a variational method for the existence of the direct problem. 
In the inverse problem, a modified linear sampling method, originated from a factorization of the chiral far field operator, has been employed.

In this work, the inverse scattering problem of specifying the shape and the surface impedance of a buried coated scattering object in a chiral environment is studied. A qualitative method [10], which is based on the chiral reciprocity gap operator, is used in Reference [11]. In fact, this procedure is a modified type of the linear sampling method (LSM). The classical LSM, which was first established by Colton and Kirsch [12], is simple, relatively quick and does not need any a priori information of the material parameters of the scattering object. However, in the electromagnetic imaging of a buried object via LSM, the computation of the Green's function of the background material is necessary. Sometimes, this computation is practically impossible. The reciprocity gap functional method helps us to overcome this difficulty. The combination of the LSM and the reciprocity gap functional method was established by Colton and Haddar in Reference [13] for acoustic waves and by Cakoni, Fares, and Haddar in Reference [14] for electromagnetic waves. In Reference [15], a reciprocity gap functional for elastic waves has been used for solving an inverse mixed impedance scattering problem. In Reference [11], a reciprocity gap functional for chiral media has been defined, in order for an inverse scattering problem for a perfect conductor to be solved.

The present paper extends this method to study inverse scattering problems for buried partially coated objects in a chiral environment. In Reference [16], the shape and the surface impedance of a buried coated scattering object have been determined. In Reference [17], the same method has been applied to solve an electromagnetic inverse scattering problem for a partially coated anisotropic dielectric, which is in the inner of the earth. Using this method in Reference [18], an inverse electromagnetic scattering problem for a perfectly conducting cavity, using measurements from the interior, has been solved. In Reference [19], an interior inverse acoustic scattering problem for a cavity with an inhomogeneous medium inside has been studied. The same method has been employed in Reference [20] in order for a sound field to be reconstructed in a spherical harmonic domain. In Reference [21], the reciprocity gap functional method is applied to calculate the boundary and the permittivity of the scattering object in radar imaging. Recently, the reciprocity gap functional method has been employed in order to study an inverse scattering problem in electrical tomography [22], in seismology [23] and in source identification [24]. For more details on the linear sampling and reciprocity gap functional method, we refer to Reference [12], while, for general aspect in scattering theory, we refer to Reference $[25,26]$.

In Section 2 of this paper, the electromagnetic waves in chiral media are described and the chiral mixed impedance scattering problem is formulated. In Section 3, the chiral reciprocity gap operator is defined, proved that it is injective and it has a dense range. In Section 4, the main result of the paper is proved. In Section 5, the surface impedance is determined. Finally, a conclusion is given in Section 6.

\section{Electromagnetic Waves in Chiral Media}

We consider the scattering of a time-harmonic electromagnetic wave by an object embedded in a chiral medium. The Drude-Born-Fedorov constitutive relations [6] are employed:

$$
\mathcal{D}=\varepsilon(\mathcal{E}+\beta \nabla \times \mathcal{E}), \quad \mathcal{B}=\mu(\mathcal{H}+\beta \nabla \times \mathcal{H}),
$$

where $\mathcal{E}, \mathcal{H}$ are the electric and magnetic fields, $\mathcal{D}$ the electric displacement, $\mathcal{B}$ the magnetic induction, $\beta$ is the chirality measure, $\varepsilon$ the electric permittivity, and $\mu$ the magnetic permeability. Then, applying the source-free Maxwell curl postulates:

$$
\nabla \times \mathcal{E}-i \omega \mathcal{B}=0, \quad \nabla \times \mathcal{H}+i \omega \mathcal{D}=0,
$$

where $\omega$ is the angular frequency, we get the following relations: 


$$
\begin{aligned}
& \nabla \times \mathcal{E}=\beta \gamma^{2} \mathcal{E}+i \omega \mu\left(\frac{\gamma}{k}\right)^{2} \mathcal{H}, \\
& \nabla \times \mathcal{H}=\beta \gamma^{2} \mathcal{H}-i \omega \mathcal{E}\left(\frac{\gamma}{k}\right)^{2} \mathcal{E},
\end{aligned}
$$

where $k^{2}=\omega^{2} \varepsilon \mu$ and $\gamma^{2}=k^{2}\left(1-\beta^{2} k^{2}\right)^{-1}$. We point out that $k$ is not a wave number and does not have any particular physical significance. We assume that the physical parameters $\beta, \varepsilon, \mu$ are positive constants and $k \beta<1$, (Reference [6] (p. 87)). The fields $\mathcal{E}$ and $\mathcal{H}$ satisfy:

$$
\nabla \cdot \mathcal{E}=\nabla \cdot \mathcal{H}=0 .
$$

We eliminate the magnetic field $\mathcal{H}$ in Equations (1) and (2) and obtain

$$
\nabla \times \nabla \times \mathcal{E}-2 \beta \gamma^{2} \nabla \times \mathcal{E}-\gamma^{2} \mathcal{E}=0 .
$$

In isotropic homogeneous chiral media, the electric and magnetic fields are composed of Left Circularly Polarized (LCP) and Right Circularly Polarized (RCP) waves with different phase speeds. So, for $\mathcal{E}$ and $\mathcal{H}$, we make use of the Bohren decomposition into Beltrami fields $Q_{L}$ and $Q_{R}$ [5], and we get

$$
\mathcal{E}=Q_{L}+Q_{R}, \quad \mathcal{H}=-i \sqrt{\frac{\varepsilon}{\mu}}\left(Q_{L}-Q_{R}\right)
$$

and hence

$$
Q_{L}=\frac{1}{2}\left(\mathcal{E}+i \sqrt{\frac{\mu}{\varepsilon}} \mathcal{H}\right), \quad Q_{R}=\frac{1}{2}\left(\mathcal{E}-i \sqrt{\frac{\mu}{\varepsilon}} \mathcal{H}\right) .
$$

The Beltrami fields satisfy the differential equations:

$$
\nabla \times Q_{L}=\gamma_{L} Q_{L}, \quad \nabla \times Q_{R}=-\gamma_{R} Q_{R},
$$

which show that the homogeneous isotropic chiral media are circularly birefringent. The wave numbers $\gamma_{L}$ and $\gamma_{R}$ for the LCP and RCP Beltrami fields, respectively, are given by:

$$
\gamma_{L}=k(1-k \beta)^{-1}, \quad \gamma_{R}=k(1+k \beta)^{-1}
$$

and satisfy:

$$
\gamma_{L}+\gamma_{R}=\frac{2 \gamma^{2}}{k}, \quad \gamma_{L}-\gamma_{R}=2 \beta \gamma^{2}, \quad \gamma_{L} \gamma_{R}=\gamma^{2} .
$$

For further information on the physical background for chiral media, we refer to Reference $[5,6,27]$.

We assume that a scatterer $D$ with $C^{2}$-boundary, $\Gamma=\partial D$ is embedded in a piecewise isotropic homogeneous chiral material with $\mathbb{R}^{3} \backslash \bar{D}$ to be connected. It is assumed that $\Gamma$ is divided into two open sets $\Gamma_{D}$ and $\Gamma_{I}$, such that $\Gamma_{D} \cap \Gamma_{I}=\varnothing$ and $\bar{\Gamma}_{D} \cup \bar{\Gamma}_{I}=\Gamma$. On $\Gamma_{D}$ (Dirichlet part), a perfectly conducting boundary condition is satisfied and $\Gamma_{I}$ (impedance part) is covered from a very thin dielectric layer. We consider $\Omega$ to be a bounded domain, which contains $\bar{D}$, with $C^{2}$ - boundary $\partial \Omega$. Let $\beta_{b}, \varepsilon_{b}$ and $\mu_{b}$ be the chirality, the electric permittivity, and the magnetic permeability, respectively, that characterize the medium $\Omega \backslash \bar{D}$, which will be referred to as the background medium. In addition, let $\beta_{0}, \varepsilon_{0}$ and $\mu_{0}$ be the corresponding parameters in the exterior $\mathbb{R}^{3} \backslash \bar{\Omega}$ of $\Omega$. We suppose that the physical parameters are positive constants. Finally, $v$ denotes the outward normal unit vector on the corresponding surface. 
The incident field is a chiral electric dipole with polarization $p \in \mathbb{R}^{3}$ located at $x_{0}$ in a chiral environment. We assume that $x_{0}$ lies on an auxiliary close surface $\Lambda$ contained in $\mathbb{R}^{3} \backslash \bar{\Omega}$. The electric incident field in a chiral medium is given by the formula $[5,6]$ :

$$
\begin{array}{r}
E_{x_{0}}\left(x, p, \gamma_{0}\right)=\frac{k_{0}}{2 \gamma_{0}^{2}} p \cdot\left\{\left(\gamma_{0 L} \widetilde{I}+\frac{1}{\gamma_{0 L}} \nabla \nabla+\nabla \times \widetilde{I}\right) \frac{e^{i \gamma_{0 L}\left|x-x_{0}\right|}}{4 \pi\left|x-x_{0}\right|}\right. \\
\left.+\left(\gamma_{0 R} \widetilde{I}+\frac{1}{\gamma_{0 R}} \nabla \nabla-\nabla \times \widetilde{I}\right) \frac{e^{i \gamma_{0 R}\left|x-x_{0}\right|}}{4 \pi\left|x-x_{0}\right|}\right\},
\end{array}
$$

where $\widetilde{I}$ is the identity dyadic in $\mathbb{R}^{3}$, and $\gamma_{0 L}, \gamma_{0 R}$ are the wave numbers for the LCP and RCP Beltrami fields, respectively, in $\mathbb{R}^{3} \backslash \bar{\Omega}$ with

$$
\gamma_{0 L}=k_{0}\left(1-k_{0} \beta_{0}\right)^{-1}, \quad \gamma_{0 R}=k_{0}\left(1+k_{0} \beta_{0}\right)^{-1},
$$

where $\gamma_{0}^{2}=\gamma_{0 L} \gamma_{0 R}, k_{0}^{2} \equiv k^{2}=\omega^{2} \varepsilon_{0} \mu_{0}$.

The incident on the scatterer $D$ electric wave $E^{i}$ has the form:

$$
E^{i}(x) \equiv E_{x_{0}}^{i}(x, p)=E_{x_{0}}\left(x, p, \gamma_{0}\right)+E_{x_{0}}^{s, b}(x, p),
$$

where $E_{x_{0}}^{s, b}(x, p)$ is the scattered field due to the background material. In addition, the wave $E^{i}$ in $\Omega \backslash \bar{D}$ is given by:

$$
E^{i}(x) \equiv E_{x_{0}}^{i}(x, p)=p \cdot \widetilde{B}\left(x, x_{0}\right),
$$

where $\widetilde{B}\left(x, x_{0}\right)$ is the dyadic Green's function of the chiral background material. If $\eta(x)=\eta_{b}=\left(\varepsilon_{b} \mu_{b}\right)\left(\varepsilon_{0} \mu_{0}\right)^{-1}$ for $x \in \Omega \backslash \bar{D}, \eta(x)=1$ for $x \in \mathbb{R}^{3} \backslash \bar{\Omega}, \beta(x)=\beta_{b}$ for $x \in \Omega \backslash \bar{D}$ and $\beta(x)=\beta_{0}$ for $x \in \mathbb{R}^{3} \backslash \bar{\Omega}$, then $\widetilde{B}\left(x, x_{0}\right)$ satisfies the equation:

$$
\left(k^{-2}-\eta(x) \beta^{2}(x)\right) \nabla \times \nabla \times \widetilde{B}\left(x, x_{0}\right)-2 \beta(x) \eta(x) \nabla \times \widetilde{B}\left(x, x_{0}\right)-\eta(x) \widetilde{B}\left(x, x_{0}\right)=\widetilde{I} \delta\left(x-x_{0}\right),
$$

with respect to $x$. Let $E^{i}$ be the incident on $D$ electric field and $E^{S} \equiv E_{x_{0}}^{S, D}(\cdot, p)$ be the corresponding scattered field. Then, the total electric field $E$ is given by $E=E^{i}+E^{s}$ and is the solution of the mixed impedance scattering problem:

$$
\begin{aligned}
& \left(k^{-2}-\eta(x) \beta^{2}(x)\right) \nabla \times \nabla \times E-2 \beta(x) \eta(x) \nabla \times E-\eta(x) E=0 \text { in } \mathbb{R}^{3} \backslash\left(\bar{D} \cup\left\{x_{0}\right\}\right), \\
& v \times E=0 \text { on } \Gamma_{D} \text {, } \\
& v \times \nabla \times E-i \frac{\gamma_{b}^{2} \lambda}{k_{b}}(v \times E) \times v-\beta_{b} \gamma_{b}^{2} v \times E=0 \text { on } \Gamma_{I} \text {, } \\
& \hat{x} \times \nabla \times E^{S}-\beta_{0} \gamma_{0}^{2} \hat{x} \times E^{S}+i \frac{\gamma_{0}^{2}}{k_{0}} E^{S}=o\left(\frac{1}{|x|}\right),|x| \rightarrow \infty \\
& \text { uniformly in all directions } \hat{x}=\frac{x}{|x|} \in S^{2} \text {, }
\end{aligned}
$$

where $S^{2}$ is the unit sphere in $\mathbb{R}^{3}, k_{b}=\omega \sqrt{\varepsilon_{b} \mu_{b}}, \gamma_{b}^{2}=\gamma_{b_{L}} \gamma_{b_{R}}$, with $\gamma_{b_{L}}=k_{b}\left(1-k_{b} \gamma_{b}\right)^{-1}$ and $\gamma_{b_{R}}=k_{b}\left(1+k_{b} \gamma_{b}\right)^{-1}$.

The direct scattering problem can be studied as in Reference [9]. The uniqueness of solution has been proved via the Beltrami fields, while, for the existence of solution, the variational method has been employed, using a Calderon type operator [28] for chiral media. The corresponding inverse scattering problem is the determination of the unknown boundary of $D$ and the evaluation of surface impedance $\lambda$ from the information of the tangential components $v \times E$ and $v \times H$ on the boundary $\partial \Omega$ for all points $x_{0} \in \Lambda$. In chiral media, a Stratton-Chu type exterior integral representation for a radiating solution of Equation (3) is the following: 


$$
\begin{aligned}
E^{S}(r)= & -2 \beta \gamma^{2} \int_{S} \widetilde{B}\left(r, r^{\prime}\right) \cdot\left[v \times E^{S}\left(r^{\prime}\right)\right] d s\left(r^{\prime}\right) \\
& +\int_{S}\left\{\widetilde{B}\left(r, r^{\prime}\right) \cdot\left[v \times \nabla \times E^{S}\left(r^{\prime}\right)\right]+\left[\nabla_{r} \times \widetilde{B}\left(r, r^{\prime}\right)\right] \cdot\left[v \times E^{S}\left(r^{\prime}\right)\right]\right\} d s\left(r^{\prime}\right) .
\end{aligned}
$$

We define the function spaces:

$$
\begin{gathered}
H(\text { curl }, D)=\left\{u \in\left(L^{2}(D)\right)^{3}: \nabla \times u \in\left(L^{2}(D)\right)^{3}\right\}, \\
H_{0}\left(\text { curl }, B_{R}\right)=\left\{u \in H\left(\text { curl, } B_{R}\right): v \times\left. u\right|_{\partial B_{R}}=0\right\},
\end{gathered}
$$

where $B_{R}$ is a ball of radius $R$ containing $D$, as well as

$$
\begin{gathered}
\mathbb{H}(\Omega)=\left\{u \in H(\operatorname{curl}, \Omega): \nabla \times \nabla \times u-2 \beta_{b} \gamma_{b}^{2} \nabla \times u-\gamma_{b}^{2} u=0\right\} \\
L_{t}^{2}(\partial D)=\left\{u \in\left(L^{2}(\partial D)\right)^{3}: v \cdot u=0 \text { on } \partial D\right\} \\
L_{t}^{2}\left(\Gamma_{I}\right)=\left\{\left.u\right|_{\Gamma_{I}}: u \in L_{t}^{2}(\partial D)\right\} \\
X\left(D, \Gamma_{I}\right)=\left\{u \in H(\text { curl }, D): v \times\left. u\right|_{\Gamma_{I}} \in L_{t}^{2}\left(\Gamma_{I}\right)\right\}
\end{gathered}
$$

The space $X\left(D, \Gamma_{I}\right)$ is equipped with the norm

$$
\|u\|_{X\left(D, \Gamma_{I}\right)}^{2}=\|u\|_{H(c u r l, D)}^{2}+\|v \times u\|_{L_{t}^{2}\left(\Gamma_{I}\right)}^{2} .
$$

For the trace $v \times u$ of $u \in H($ curl, D), we have

$$
H_{d i v}^{-\frac{1}{2}}(\partial D)=\left\{u \in\left(H^{-\frac{1}{2}}(\partial D)\right)^{3}: v \cdot u=0, \operatorname{div}_{\partial D} u \in H^{-\frac{1}{2}}(\partial D)\right\},
$$

and for $(v \times u) \times v$ of $u \in H(c u r l, D)$

$$
H_{\text {curl }}^{-\frac{1}{2}}(\partial D)=\left\{u \in\left(H^{-\frac{1}{2}}(\partial D)\right)^{3}: v \cdot u=0, \operatorname{curl}_{\partial D} u \in H^{-\frac{1}{2}}(\partial D)\right\} .
$$

The trace space of $X\left(D, \Gamma_{I}\right)$ on $\Gamma_{D}$ is defined by:

$$
Y\left(\Gamma_{D}\right)=\left\{h \in\left(H^{-\frac{1}{2}}\left(\Gamma_{D}\right)\right)^{3}: \exists u \in H_{0}\left(\text { curl, } B_{R}\right), v \times\left. u\right|_{\Gamma_{I}} \in L_{t}^{2}\left(\Gamma_{I}\right), h=v \times\left. u\right|_{\Gamma_{D}}\right\} .
$$

Finally, for the exterior domain $\mathbb{R}^{3} \backslash \bar{D}$, we define the spaces $H_{l o c}\left(\right.$ curl, $\left.\mathbb{R}^{3} \backslash \bar{D}\right)$ and $H_{\text {loc }}\left(\mathbb{R}^{3} \backslash \bar{D}, \Gamma_{I}\right)$ considering the domain $\left(\mathbb{R}^{3} \backslash \bar{D}\right) \cap B_{R}$.

The exterior mixed impedance boundary value problem in chiral media is the following problem: Let $f \in Y\left(\Gamma_{D}\right)$ and $h \in L_{t}^{2}\left(\Gamma_{I}\right)$, find $E \in X\left(D, \Gamma_{I}\right)$ such that:

$$
\begin{gathered}
\left(k^{-2}-\eta(x) \beta^{2}(x)\right) \nabla \times \nabla \times E-2 \beta(x) \eta(x) \nabla \times E-\eta(x) E=0 \text { in } \mathbb{R}^{3} \backslash \bar{D}, \\
v \times E=f \text { on } \Gamma_{D}, \\
v \times \nabla \times E-i \frac{\gamma_{b}^{2} \lambda}{k_{b}}(v \times E) \times v-\beta_{b} \gamma_{b}^{2} v \times E=h \text { on } \Gamma_{I}, \\
\hat{x} \times \nabla \times E-\beta_{0} \gamma_{0}^{2} \hat{x} \times E+i \frac{\gamma_{0}^{2}}{k_{0}} E=o\left(\frac{1}{|x|}\right),|x| \rightarrow \infty \\
\text { uniformly in all directions } \hat{x}=\frac{x}{|x|} \in S^{2},
\end{gathered}
$$

If $f=-v \times E^{i}$ and $h=-v \times \nabla \times E^{i}+i \frac{\gamma_{b}^{2} \lambda}{k_{b}}\left(v \times E^{i}\right) \times v+\beta_{b} \gamma_{b}^{2} v \times E^{i}$, then the problem (11)-(14) is the mixed impedance scattering problem (6)-(9). 
Let $z \in D$ and $E^{z} \in H($ curl, $D)$. We consider the following chiral interior mixed impedance boundary value problem corresponding to (6)-(9); given $f \in Y\left(\Gamma_{D}\right)$ and $h \in L_{t}^{2}\left(\Gamma_{I}\right)$, we find $E^{z} \in X\left(D, \Gamma_{I}\right)$ such that:

$$
\begin{gathered}
\nabla \times \nabla \times E^{z}-2 \beta_{b} \gamma_{b}^{2} \nabla \times E^{z}-\gamma_{b}^{2} E^{z}=0 \text { in } D, \\
v \times E^{z}=f \text { on } \Gamma_{D}, \\
v \times \nabla \times E^{z}-i \frac{\gamma_{b}^{2} \lambda}{k_{b}}\left(v \times E^{z}\right) \times v+\beta_{b} \gamma_{b}^{2} v \times E^{z}=h \text { on } \Gamma_{I} .
\end{gathered}
$$

The values of parameter $k$ for which the corresponding homogeneous interior mixed impedance scattering problem admits a nontrivial solution will be referred to as chiral Maxwell eigenvalues for $D$. This problem in the achiral case has been solved in Reference [29]. A similar scattering problem for a mixed impedance screen has been studied in Reference [9]. In particular, a Calderon type operator for chiral media and a variational method have been employed to prove uniqueness and existence of solution. The present scattering problem is to find the shape of $D$ and the surface impedance $\lambda$ from the knowledge of electric and magnetic fields on $\partial \Omega$. In what follows, a brief description of the solvability of the interior mixed impedance problem (15)-(17) is given.

For the uniqueness of (15)-(17), we consider the corresponding homogeneous problem $(f=h=0)$, and we multiply (15) with $\bar{E}$ (complex conjugate of $E$ ) and integrate over $D$. Taking into account the boundary conditions we get

$$
\int_{D}\left[|\nabla \times E|^{2}-\gamma_{b}^{2}|E|^{2}-2 \beta_{b} \gamma_{b}^{2} \operatorname{Re}(E \cdot \nabla \times \bar{E})\right] d v+i \frac{\gamma_{b}^{2} \lambda}{k_{b}} \int_{\Gamma_{I}}\left|E_{T}\right|^{2} d s=0,
$$

where $E_{T}=(v \times E) \times v$ is the tangential component of $E$. From (18), taking the imaginary part and using the unique continuation principle as in Reference [12,29], we conclude that $E=0$ in $D$. For the existence, we consider the variational formulation for the problem (15)-(17). For all test functions $\phi \in \widetilde{X}$ with

$$
\widetilde{X}=\left\{u \in H(\operatorname{curl}, D): v \times\left. u\right|_{\Gamma_{D}}=0, v \times\left. u\right|_{\Gamma_{I}} \in L_{t}^{2}\left(\Gamma_{I}\right)\right\},
$$

we have

$$
\begin{aligned}
& \int_{D}\left[\nabla \times E \cdot \nabla \times \bar{\phi}-\gamma^{2} E \cdot \bar{\phi}-2 \beta \gamma^{2} \operatorname{Re}(\bar{\phi} \cdot \nabla \times E)\right] d v+\frac{i \gamma^{2} \lambda}{k} \int_{\Gamma_{I}} E_{T} \cdot \bar{\phi}_{T} d s \\
& =-\int_{\Gamma_{I}} h \cdot \bar{\phi}_{T} d s .
\end{aligned}
$$

We look for solution $E$ of the form $E=W+U$, where $U \in X\left(D, \Gamma_{I}\right)$ with $v \times\left. U\right|_{\Gamma_{D}}=f$, which there exists from the definition of $Y\left(\Gamma_{D}\right)$. Substituting in (19), we take:

$$
a(W, \phi)=\langle h, \phi\rangle-a(U, \phi),
$$

where

$$
a(u, \psi)=(\nabla \times u, \nabla \times \psi)-\gamma^{2}(u, \phi)-2 \beta \gamma^{2} \operatorname{Re}(\nabla \times u, \phi)+\frac{i \gamma^{2} \lambda}{k}\left\langle u_{T}, \psi_{T}\right\rangle .
$$

In $(21),(\cdot, \cdot)$ denotes the $L^{2}(D)$ scalar product and $\langle\cdot, \cdot\rangle$ the $L_{t}^{2}\left(\Gamma_{I}\right)$ product. Equation (20) has been studied in Reference $[28,29]$ for the achiral case. With a similar process for the chiral case, the following theorem is proved.

Theorem 1. If $\Gamma_{I} \neq \varnothing$ then the chiral interior partially coated problem (15)-(17) has a unique solution. 


\section{The Chiral Reciprocity Gap Operator}

The reciprocity gap operator for electromagnetic scattering in chiral media has been defined in Reference [11], in order to study an inverse scattering problem for a perfectly conducting obstacle.

Let $E=E_{x_{0}}(\cdot, p)$ be the solution of the scattering problem (6)-(9). The chiral reciprocity gap functional is defined by

$$
\mathcal{R}(E, W)=\int_{\partial \Omega}[(v \times E) \cdot \nabla \times W-(v \times W) \cdot \nabla \times E] d s-2 \beta_{b} \gamma_{b}^{2} \int_{\partial \Omega}[(v \times E) \cdot W] d s,
$$

where $W \in H(\mathrm{curl}, \Omega)$ and the integrals are interpreted in the sense of the duality between $H_{\text {div }}^{-\frac{1}{2}}(\partial D), H_{\text {curl }}^{-\frac{1}{2}}(\partial D)$. In particular, if $W \in \mathbb{H}(\Omega) \subset H($ curl,$\Omega)$, then the chiral reciprocity gap functional can be seen as an integral operator $R: \mathbb{H}(\Omega) \rightarrow L_{t}^{2}(\Lambda)$, given by:

$$
R(W)\left(x_{0}\right)=\mathcal{R}\left(E_{x_{0}}\left(\cdot, p\left(x_{0}\right)\right), W\right) p\left(x_{0}\right), \quad x_{0} \in \Lambda .
$$

The reciprocity gap functional method is based on the solvability of an integral equation for $\mathcal{R}$, which contains an appropriate family of solutions in $\mathbb{H}(\Omega)$. Usually, we use a set of either single layer potentials or Hergotz wave functions. Here, for the determination of the boundary of $D$, chiral Herglotz wave functions will be employed, because these functions satisfy density properties which will be used later. In Reference [2], the electric $\mathcal{E}_{g}$ and magnetic $\mathcal{H}_{g}$ chiral Herglotz wave functions have been defined and are given by

$$
\mathcal{E}_{g}=\mathcal{E}_{g_{L}}+\mathcal{E}_{g_{R}}, \quad \mathcal{H}_{g}=-i \sqrt{\frac{\varepsilon}{\mu}}\left(\mathcal{E}_{g_{L}}-\mathcal{E}_{g_{R}}\right),
$$

where

$$
\begin{aligned}
\mathcal{E}_{g_{L}}(x) & =\int_{S^{2}} g_{L}\left(\hat{d}_{L}\right) e^{i \gamma_{L} \hat{d}_{L} \cdot x} d s\left(\hat{d}_{L}\right), \\
\mathcal{E}_{g_{R}}(x) & =\int_{S^{2}} g_{R}\left(\hat{d}_{R}\right) e^{i \gamma_{R} \hat{d}_{R} \cdot x} d s\left(\hat{d}_{R}\right),
\end{aligned}
$$

are the LCP and the RCP Beltrami Herglotz fields, with kernels $g_{L}$ and $g_{R}$, respectively, and $\hat{d}_{L}, \hat{d}_{R} \in S^{2}$. In particular, for the kernels, we have $g_{A}: S^{2} \rightarrow T_{A}^{2}\left(S^{2}\right), A=L, R$, where

$$
\begin{aligned}
& T_{L}^{2}\left(S^{2}\right)=\left\{b_{L} \in\left(L^{2}\left(S^{2}\right)\right)^{3}: v \cdot b_{L}=0, v \times b_{L}=-i b_{L}\right\}, \\
& T_{R}^{2}\left(S^{2}\right)=\left\{b_{R} \in\left(L^{2}\left(S^{2}\right)\right)^{3}: v \cdot b_{R}=0, v \times b_{R}=i b_{R}\right\} .
\end{aligned}
$$

In addition, we define the following space:

$$
T_{L R}^{2}\left(S^{2}\right)=\left\{b=b_{L}+b_{R}: b_{L} \in T_{L}^{2}\left(S^{2}\right), b_{R} \in T_{R}^{2}\left(S^{2}\right)\right\},
$$

with the inner product:

$$
<b, h>_{T_{L R}^{2}\left(S^{2}\right)}=\left(b_{L}, h_{L}\right)_{T_{L}^{2}\left(S^{2}\right)}+\left(b_{R}, h_{R}\right)_{T_{R}^{2}\left(S^{2}\right)},
$$

where $b_{A}, h_{A}, A=L, R$, are the Beltrami fields of $b$ and $h$, respectively, and $\left(b_{A}, h_{A}\right)_{T_{A}^{2}\left(S^{2}\right)}=\int_{S^{2}} b_{A} \cdot \bar{h}_{A} d s$ [2]. Let

$$
\begin{aligned}
E_{z}\left(x, q, \gamma_{b}\right)=\frac{k_{b}}{2 \gamma_{b}^{2}} q \cdot\left\{\left(\gamma_{b L} \widetilde{I}+\frac{1}{\gamma_{b L}} \nabla \nabla+\nabla \times \widetilde{I}\right) \frac{e^{i \gamma_{b L}|x-z|}}{4 \pi|x-z|}\right. \\
\left.+\left(\gamma_{b R} \widetilde{I}+\frac{1}{\gamma_{b R}} \nabla \nabla-\nabla \times \widetilde{I}\right) \frac{e^{i \gamma_{b R}|x-z|}}{4 \pi|x-z|}\right\},
\end{aligned}
$$


be the electric dipole with polarization $q \in \mathbb{R}^{3}$ located at $z$ in a chiral medium. We study the solvability of the integral equation:

$$
\mathcal{R}\left(E, \mathcal{E}_{g}\right)=\mathcal{R}\left(E, E_{z}\left(\cdot, q, \gamma_{b}\right)\right),
$$

with respect to $g$ in $T_{L R}^{2}\left(S^{2}\right)$.

We will prove that the operator $R$, under appropriate conditions, is injective and has dense range.

Lemma 1. If $\Gamma_{I}$ is not empty then the operator $R: \mathbb{H}(\Omega) \rightarrow L_{t}^{2}(\Lambda)$, defined by (23) is injective.

Proof. We assume that $R W=0$. Then, $\mathcal{R}\left(E_{x_{0}}(\cdot, p), W\right)=0$ for all $x_{0} \in \Lambda$ and $p \in \mathbb{R}^{3}$. On (22), we apply the second vector Green's theorem for the first integral, Gauss' theorem for the second integral for $E, W$ in $\Omega \backslash \bar{D}$, which are both solutions of (15), we use the boundary conditions on $\partial D$ to take

$$
\begin{aligned}
0= & \int_{\partial D}[(v \times E) \cdot \nabla \times W-(v \times W) \cdot \nabla \times E] d s-2 \beta_{b} \gamma_{b}^{2} \int_{\partial D}(v \times E) \cdot W d s \\
= & -\int_{\Gamma_{D}}(v \times W) \cdot \nabla \times E d s \\
& -\int_{\Gamma_{I}} E \cdot\left[v \times \nabla \times W-i \frac{\gamma_{b}^{2} \lambda}{k_{b}}(v \times W) \times v-\beta_{b} \gamma_{b}^{2}(v \times W)\right] d s .
\end{aligned}
$$

Let $\breve{E}$ be the unique solution of the boundary value problem:

$$
\begin{gathered}
\left(k^{-2}-\beta(x)^{2} \eta(x)\right) \nabla \times \nabla \times \breve{E}-2 \beta(x) \eta(x) \nabla \times \breve{E}-\eta(x) \breve{E}=0 \text { in } \mathbb{R}^{3} \backslash \bar{D}, \\
v \times(\breve{E}-W)=0 \text { on } \Gamma_{D}, \\
v \times \nabla \times(\breve{E}-W)=i \frac{\gamma_{b}^{2} \lambda}{k_{b}}[v \times(\breve{E}-W)] \times v+\beta_{b} \gamma_{b}^{2} v \times(\breve{E}-W) \text { on } \Gamma_{I}, \\
\frac{x}{|x|} \times \nabla \times \breve{E}-\beta_{0} \gamma_{0}^{2} \frac{x}{|x|} \times \breve{E}+\frac{i \gamma_{0}^{2}}{k_{0}} \breve{E}=o\left(\frac{1}{|x|}\right),|x| \rightarrow \infty \\
\text { uniformly in all directions of } \frac{x}{|x|} \in S^{2} .
\end{gathered}
$$

Substituting $v \times W$ and $v \times \nabla \times W$, from (29) and (30) into (27), we take

$$
\begin{aligned}
0= & -\int_{\Gamma_{D}}(v \times \breve{E}) \cdot \nabla \times E d s \\
& -\int_{\Gamma_{I}} E \cdot\left[v \times \nabla \times \breve{E}-i \frac{\gamma_{b}^{2} \lambda}{k_{b}}(v \times \breve{E}) \times v-\beta_{b} \gamma_{b}^{2}(v \times \breve{E})\right] d s .
\end{aligned}
$$

The total electric field $E$ is given by:

$$
E=p \cdot \widetilde{B}\left(\cdot, x_{0}\right)+E^{s} .
$$

Hence, using (32) and the boundary conditions (7) and (8), we get

$$
\begin{aligned}
0= & \int_{\partial D}\left[\left(v \times\left(p \cdot \widetilde{B}\left(\cdot, x_{0}\right)+E^{s}\right)\right) \cdot \nabla \times \breve{E}-(v \times \breve{E}) \cdot \nabla \times\left(p \cdot \widetilde{B}\left(\cdot, x_{0}\right)+E^{s}\right)\right] d s \\
& -2 \beta_{b} \gamma_{b}^{2} \int_{\partial D}\left[\left(v \times\left(p \cdot \widetilde{B}\left(\cdot, x_{0}\right)+E^{s}\right)\right) \cdot \breve{E}\right] d s
\end{aligned}
$$


and taking into account that the fields $\breve{E}$ and $E^{S}$ are both radiating solutions of (28), we have:

$$
\begin{aligned}
-p \cdot\left\{\int _ { \partial D } \left[\widetilde{B}\left(\cdot, x_{0}\right) \cdot(\right.\right. & \left.\times \nabla \times \breve{E})+\left(\nabla \times \widetilde{B}\left(\cdot, x_{0}\right)\right) \cdot(v \times \breve{E})\right] d s \\
& \left.-2 \beta_{b} \gamma_{b}^{2} \int_{\partial D} \widetilde{B}\left(\cdot, x_{0}\right) \cdot(v \times \breve{E}) d s\right\}=0 .
\end{aligned}
$$

From the Stratton-Chu type formula (10) for chiral media, we take

$$
p \cdot \breve{E}\left(x_{0}\right)=0,
$$

for arbitrary polarization $p$, and therefore $v \times \breve{E}\left(x_{0}\right)=0$ for $x_{0} \in \Lambda$. Then, by the uniqueness of the electromagnetic scattering in a chiral environment for a perfect conductor [1,3], we conclude that $\breve{E}=0$ outside the surface $\Lambda$. Applying unique continuation, we have $\breve{E}=0$ in the domain between the boundary $\partial D$ and the surface $\Lambda$. Therefore,

$$
\begin{gathered}
v \times W=0 \text { on } \Gamma_{D}, \\
v \times \nabla \times W-i \frac{\gamma_{b}^{2} \lambda}{k_{b}}(v \times W) \times v-\beta_{b} \gamma_{b}^{2} v \times W=0 \text { on } \Gamma_{I}
\end{gathered}
$$

and using the uniqueness of the interior partially coated chiral electromagnetic problem for $W$, implying $W=0$.

Lemma 2. If $\Gamma_{I}$ is not empty then the operator $R: \mathbb{H}(\Omega) \rightarrow L_{t}^{2}(\Lambda)$ defined by (23) has dense range.

Proof. Let $q \in L_{t}^{2}(\Lambda)$, such that $(R W, q)_{L_{t}^{2}(\Lambda)}=0$ for all $W \in \mathbb{H}(\Omega)$. We will prove that $q=0$. In view of the bilinearity of functional $\mathcal{R}$ and the definition of operator $R$, we get

$$
(R W, q)_{L_{t}^{2}(\Lambda)}=\int_{\Lambda} \mathcal{R}\left(E_{x_{0}}\left(\cdot, \alpha\left(x_{0}\right)\right), W\right) d s,
$$

where $\alpha=(p \cdot q) p$. If we define

$$
\mathcal{E}(x)=\int_{\Lambda} E_{x_{0}}\left(x, \alpha\left(x_{0}\right)\right) d s\left(x_{0}\right),
$$

then, from (22) and the assumption for $q$, we have that

$$
\mathcal{R}(\mathcal{E}, W)=0 .
$$

Using Green's and Gauss' theorems for $W, \mathcal{E}$ in $\Omega \backslash \bar{D}$ as in Lemma 1 and taking into account the boundary conditions on $\partial D$, we conclude that

$$
\begin{aligned}
\mathcal{R}(\mathcal{E}, W)= & -\int_{\Gamma_{D}}(v \times W) \cdot \nabla \times \mathcal{E} d s \\
& -\int_{\Gamma_{I}} \mathcal{E} \cdot\left[v \times \nabla \times W-i \frac{\gamma_{b}^{2} \lambda}{k_{b}}(v \times W) \times v-\beta_{b} \gamma_{b}^{2}(v \times W)\right] d s=0,
\end{aligned}
$$

for all $W \in \mathbb{H}(\Omega)$. The density of the chiral Herglotz wave functions has been used in order to prove that the set $\left\{v \times\left. W\right|_{\Gamma_{D}}, v \times \nabla \times W-i \frac{\gamma_{b}^{2} \lambda}{k_{b}}(v \times W) \times v-\left.\beta_{b} \gamma_{b}^{2}(v \times W)\right|_{\Gamma_{I}}\right\}$ is dense in $Y\left(\Gamma_{D}\right) \times L_{t}^{2}\left(\Gamma_{I}\right)$. This follows from the fact that $\mathbb{H}(\Omega)$ contains the chiral Herglotz wave functions, given by (24) and (25) (see [2,29]), which satisfy the Equation (3) and $\nabla \times \nabla \times Q_{A}-\gamma_{A}^{2} Q_{A}=0, A=L, R$. In addition, we have taken into account that the interior mixed impedance boundary value problem (15)-(17) is well-posed. Therefore, $v \times \nabla \times \mathcal{E}=0$ and $v \times \mathcal{E}=0$ on $\partial D$. Hence, $\mathcal{E}$ has zero Cauchy data on $\partial D$ and therefore 
$\mathcal{E}=0$ in the domain between $\Lambda$ and $\partial D$. Finally, taking into account the jump relations [3] of $\nabla \times \mathcal{E}$ across $\Lambda$, we arrive at $\alpha=0$ on $\Lambda$. Therefore, $(p \cdot q) p=0$ for all $p \in L_{t}^{2}(\Lambda)$, hence $q=0$.

\section{The Reconstruction of the Shape}

These properties of the chiral reciprocity gap operator are used for the determination of the boundary of the scatterer $D$. The main result of this paper is the following theorem.

Theorem 2. Assume that $\Gamma_{I}$ is not empty.

(i) Let $z \in D$. Then, for a given $\epsilon>0$ there exists a $g_{z}^{\epsilon} \in T_{L R}^{2}\left(S^{2}\right)$ such that

$$
\left\|\mathcal{R}\left(E, \mathcal{E}_{g_{z}}\right)-\mathcal{R}\left(E, E_{z}\left(\cdot, q, \gamma_{b}\right)\right)\right\|_{L^{2}(\Lambda)}<\epsilon
$$

and the chiral Herglotz wave function $\mathcal{E}_{g \underset{z}{\epsilon}}$ converges to the solution of interior boundary value problem in $X\left(D, \Gamma_{I}\right)$ as $\epsilon \rightarrow 0$.

(ii) For a fixed $\epsilon>0$, we get

$$
\lim _{\operatorname{dist}(z, \partial D) \rightarrow 0}\left\|\mathcal{E}_{\mathcal{g}_{z}}\right\|_{X\left(D, \Gamma_{I}\right)}=\infty, \lim _{\operatorname{dist}(z, \partial D) \rightarrow 0}\left\|g_{z}^{\epsilon}\right\|_{T_{L R}^{2}\left(S^{2}\right)}=\infty .
$$

(iii) For $z \in \mathbb{R}^{3} \backslash \bar{D}$ and $\epsilon>0$, if $g_{z}^{\epsilon} \in T_{L R}^{2}\left(S^{2}\right)$ satisfies

$$
\left\|\mathcal{R}\left(E, \mathcal{E}_{g_{z}^{\epsilon}}\right)-\mathcal{R}\left(E, E_{z}\left(\cdot, q, \gamma_{b}\right)\right)\right\|_{L^{2}(\Lambda)}<\epsilon,
$$

then we have that

$$
\lim _{\epsilon \rightarrow 0}\left\|\mathcal{E}_{g_{z}}\right\|_{X\left(D, \Gamma_{I}\right)}=\infty, \lim _{\epsilon \rightarrow 0}\left\|g_{z}^{\epsilon}\right\|_{T_{L R}^{2}\left(S^{2}\right)}=\infty
$$

Proof. (i) Suppose $z \in D$. Taking into account that $E$ is the total field and $W$ and $E_{z}\left(\cdot, q, \gamma_{b}\right)$ are solutions to Equation (11) in $\Omega \backslash \bar{D}$ and using the mixed boundary conditions on $D$, we have that

$$
\mathcal{R}(E, W)-\mathcal{R}\left(E, E_{z}\left(\cdot, q, \gamma_{b}\right)\right)=-\int_{\partial D}\left[v \times W-v \times E_{z}\left(\cdot, q, \gamma_{b}\right)\right] \cdot \nabla \times E d s .
$$

Taking into account that the set of chiral Herglotz functions is dense in $\mathbb{H}(\Omega)$ with respect to the $H(\mathrm{curl}, D)$ norm and using the trace theorem it follows that for every $\epsilon>0$ there exists a chiral electric Herglotz function $\mathcal{E}_{g_{z}^{\epsilon}}$ such that: $v \times \mathcal{E}_{g_{z}^{\epsilon}}$ approximates $v \times E_{z}\left(\cdot, q, \gamma_{b}\right)$ with respect to $Y\left(\Gamma_{D}\right)$ norm, and $v \times \nabla \times \mathcal{E}_{g_{z}^{\epsilon}}-i \frac{\gamma_{b}^{2} \lambda}{k_{b}}\left(v \times \mathcal{E}_{g_{z}^{\epsilon}}\right) \times v-\beta_{b} \gamma_{b}^{2}(v \times$ $\left.\mathcal{E}_{g \bar{\varepsilon}}\right)$ approximates $v \times \nabla \times E_{z}\left(\cdot, q, \gamma_{b}\right)-i \frac{\gamma_{b}^{2} \lambda}{k_{b}}\left(v \times E_{z}\left(\cdot, q, \gamma_{b}\right)\right) \times v-\beta_{b} \gamma_{b}^{2}\left(v \times E_{z}\left(\cdot, q, \gamma_{b}\right)\right)$ with respect to $L_{t}^{2}\left(\Gamma_{I}\right)$ norm. In addition, $g_{z}^{\epsilon}$ solves by approximation the Equation (26) and $\mathcal{E}_{g_{z}^{\epsilon}}$ converges to the solution of the mixed chiral interior boundary value problem (15)-(17). (ii) Taking into account that $E_{z}\left(\cdot, q, \gamma_{b}\right)$ blows up as $z$ approaches the boundary $\partial D$ from inside, with respect to the $X\left(D, \Gamma_{I}\right)$ norm, we conclude: $\lim _{\operatorname{dist}(z, \partial D) \rightarrow 0}\left\|\mathcal{E}_{g_{z}}\right\|_{X\left(D, \Gamma_{I}\right)}=\infty$ and $\left.\lim _{\operatorname{dist}(z, \partial D) \rightarrow 0}|| g_{z}^{\epsilon}\right|_{T_{L R}^{2}\left(S^{2}\right)}=\infty$, with fixed $\epsilon>0$.

(iii) Let $z \in \Omega \backslash \bar{D}$. The total electric field $E(x) \equiv E_{x_{0}}(x, p)$, due to the incident point-source $E_{x_{0}}^{i}(x, p)$, is given by:

$$
E(x) \equiv E_{x_{0}}(x, p)=p \cdot \widetilde{B}\left(x, x_{0}\right)+E_{x_{0}}^{s}(x, p),
$$

where $E_{x_{0}}^{s}(x, p)$ is the corresponding scattered field. From (33) and the definition (22) we obtain

$$
\mathcal{R}\left(E, E_{z}\left(\cdot, q, \gamma_{b}\right)\right)=I_{1}+I_{2},
$$


where

$$
\begin{aligned}
I_{1}= & \int_{\partial \Omega}\left[\left(v \times\left(p \cdot \widetilde{B}\left(x, x_{0}\right)\right)\right) \cdot \nabla \times E_{z}\left(x, q, \gamma_{b}\right)-\left(v \times E_{z}\left(x, q, \gamma_{b}\right)\right) \cdot \nabla \times\left(p \cdot \widetilde{B}\left(x, x_{0}\right)\right)\right] d s(x) \\
& -2 \beta_{b} \gamma_{b}^{2} \int_{\partial \Omega}\left(v \times\left(p \cdot \widetilde{B}\left(x, x_{0}\right)\right)\right) \cdot E_{z}\left(x, q, \gamma_{b}\right) d s(x), \\
I_{2}= & \int_{\partial \Omega}\left[\left(v \times E_{x_{0}}^{s}(x, p)\right) \cdot \nabla \times E_{z}\left(x, q, \gamma_{b}\right)-\left(v \times E_{z}\left(x, q, \gamma_{b}\right)\right) \cdot \nabla \times E_{x_{0}}^{s}(x, p)\right] d s(x) \\
& -2 \beta_{b} \gamma_{b}^{2} \int_{\partial \Omega}\left(v \times E_{x_{0}}^{s}(x, p)\right) \cdot E_{z}\left(x, q, \gamma_{b}\right) d s(x),
\end{aligned}
$$

For $z \in \Omega \backslash \bar{D}$, the function $E_{z}\left(x, q, \gamma_{b}\right)$ is the fundamental solution of

$$
\nabla \times \nabla \times E-2 \beta_{b} \gamma_{b}^{2} \nabla \times E-\gamma_{b}^{2} E=0
$$

and $p \cdot \widetilde{B}\left(x, x_{0}\right), x \in \Omega \backslash \bar{D}$, is a solution of (34). Hence, $I_{1}$ is an integral representation Stratton-Chu type in chiral media (10) for $-p \cdot \widetilde{B}\left(z, x_{0}\right), z \in \Omega \backslash \bar{D}$. By making use of the reciprocity properties [6]

$$
\widetilde{B}\left(x, x_{0}\right)=\left[\widetilde{B}\left(x_{0}, x\right)\right]^{\top}, \nabla_{x} \times \widetilde{B}\left(x, x_{0}\right)=\left[\nabla_{x_{0}} \times \widetilde{B}\left(x_{0}, x\right)\right]^{\top},
$$

where $T$ denotes transposition, we conclude that the background dyadic Green's function solves (34) with respect to $x_{0}$. Hence, $E_{x_{0}}^{s}(x, p)$ satisfies the same equation with respect to $x_{0}$. Therefore, the integral $I_{2}$ gives a solution $W\left(x_{0}\right)$ of (34). Let $\mathcal{E}_{g_{z}}$ be a chiral electric Herglotz functions such that

$$
\left\|\mathcal{R}\left(E, \mathcal{E}_{g_{z}}\right)-\mathcal{R}\left(E, E_{z}\left(\cdot, q, \gamma_{b}\right)\right)\right\|_{L^{2}(\Lambda)}<\epsilon .
$$

From the definition (22) and the boundary conditions (7) and (8), we obtain

$$
\begin{aligned}
\mathcal{R}\left(E, \mathcal{E}_{g_{\bar{z}}}\right)= & -\int_{\Gamma_{D}}\left(v \times \mathcal{E}_{g_{\bar{z}}}\right) \cdot \nabla \times E d s \\
& -\int_{\Gamma_{I}} E \cdot\left[v \times \nabla \times \mathcal{E}_{g_{\bar{z}}}-i \frac{\gamma_{b}^{2} \lambda}{k_{b}}\left(v \times \mathcal{E}_{g_{\underline{z}}}\right) \times v-\beta_{b} \gamma_{b}^{2}\left(v \times \mathcal{E}_{g_{\bar{z}}}\right)\right] d s .
\end{aligned}
$$

Therefore

$$
\begin{aligned}
& \mathcal{R}\left(E, \mathcal{E}_{g_{z}}\right)-\mathcal{R}\left(E, E_{z}\left(\cdot, q, \gamma_{b}\right)\right)=-\int_{\Gamma_{D}}\left(v \times \mathcal{E}_{g_{z}^{\varepsilon}}\right) \cdot \nabla \times E d s \\
& -\int_{\Gamma_{I}} E \cdot\left[v \times \nabla \times \mathcal{E}_{g_{z}^{\varepsilon}}-i \frac{\gamma_{b}^{2} \lambda}{k_{b}}\left(v \times \mathcal{E}_{g_{z}^{\varepsilon}}\right) \times v-\beta_{b} \gamma_{b}^{2}\left(v \times \mathcal{E}_{g_{z}^{\varepsilon}}\right)\right] d s-W\left(x_{0}\right)+p \cdot \widetilde{B}\left(x, x_{0}\right) .
\end{aligned}
$$

We assume that $\left\|\mathcal{E}_{g_{z}}\right\|_{X\left(D, \Gamma_{I}\right)}<c$, with $c$ constant, positive and independent of $\epsilon$. Applying the trace theorem, we take the trace of $\mathcal{E}_{g_{z}}$ also bounded, with respect to the corresponding norms. Therefore, there exists a weakly convergent subfamily converging to a function $V \in X\left(D, \Gamma_{I}\right)$ as $\epsilon \rightarrow 0$. For $x_{0} \in \Lambda$, we set:

$$
\begin{aligned}
U\left(x_{0}\right)= & -\int_{\Gamma_{D}}(v \times V) \cdot \nabla \times E_{x_{0}}(\cdot, p) d s \\
& -\int_{\Gamma_{I}} E \cdot\left[v \times \nabla \times V-i \frac{\gamma_{b}^{2} \lambda}{k_{b}}(v \times V) \times v-\beta \gamma_{b}^{2}(v \times V)\right] d s .
\end{aligned}
$$

From (35) and (36), we obtain:

$$
U\left(x_{0}\right)=W\left(x_{0}\right)+p \cdot \widetilde{B}\left(z, x_{0}\right), x_{0} \in \Lambda .
$$


Taking into account that the functions $U\left(x_{0}\right)$ and $W\left(x_{0}\right)$ are radiating solutions of (34) and using the unique continuation principle, we conclude that (37) holds true in $\mathbb{R}^{3} \backslash(\bar{D} \cup\{z\})$. If we now let $x_{0} \rightarrow z$, then we arrive at a contradiction.

Remark 1. The determination of the boundary $\partial D$ of the scatterer is based on the integral Equation (26), which contains chiral Herglotz functions in $\mathbb{H}(\Omega)$. In particular, if $\mathcal{E}_{g_{z}^{e}}$ is a solution of (26), then the boundary $\partial D$ of the scatterer is reconstructed from points $z$, with $\lim _{\epsilon \rightarrow 0}\left\|g_{z}^{\epsilon}\right\|_{T_{L R}^{2}\left(S^{2}\right)}=\infty$. It is obvious that the boundary $\partial D$ cannot be found from the $\lim _{\epsilon \rightarrow 0}\left\|\mathcal{E}_{g_{z}}\right\| \|_{X\left(D, \Gamma_{I}\right)}=\infty$ since the corresponding norm is defined on the unknown scatterer D. Alternatively, one can use instead of the chiral Herglotz functions appropriate potentials [3,10].

\section{The Determination of the Surface Impedance}

Finally, after determining $D$, we will establish an expression for the surface impedance $\lambda$. In particular, we prove the following theorem.

Theorem 3. Let $E^{z}$ be the solution of (15)-(17) for a fix point $z \in D$. Then, the surface impedance $\lambda$ is given by

$$
\lambda=\frac{k_{b}}{2 \gamma_{b}^{2}} \frac{\operatorname{Im}\left(q \cdot E^{z}(z)\right)+I_{z}\left(\Omega, q, \gamma_{b}\right)}{\int_{\partial D}\left|v \times\left(E^{z}-E_{z}\left(\cdot, q, \gamma_{b}\right)\right)\right|^{2} d s}
$$

where the integral

$$
\begin{aligned}
I_{z}\left(\Omega, q, \gamma_{b}\right)= & -i \int_{\partial \Omega}\left[\left(v \times E_{z}\left(\cdot, q, \gamma_{b}\right)\right) \cdot \nabla \times \overline{E_{z}\left(\cdot, q, \gamma_{b}\right)}-\left(v \times \overline{E_{z}\left(\cdot, q, \gamma_{b}\right)}\right) \cdot \nabla \times E_{z}\left(\cdot, q, \gamma_{b}\right)\right] d s \\
& +2 i \beta_{b} \gamma_{b}^{2} \int_{\partial \Omega}\left(v \times E_{z}\left(\cdot, q, \gamma_{b}\right)\right) \cdot \overline{E_{z}\left(\cdot, q, \gamma_{b}\right)} d s
\end{aligned}
$$

is depended on $z, \Omega$ and $q$.

Proof. For a fix point $z \in D$, we consider the unique solution $E^{z}$ of the interior mixed boundary value problem (15)-(17). We define the function:

$$
U^{z}(x)=E^{z}(x)-E_{z}\left(x, q, \gamma_{b}\right), x \in D
$$

and we evaluate the integral:

$$
I=\int_{\partial D}\left[\left(v \times U^{z}\right) \cdot \nabla \times \overline{U^{z}}-\left(v \times \overline{U^{z}}\right) \cdot \nabla \times U^{z}\right] d s-2 \beta_{b} \gamma_{b}^{2} \int_{\partial D}\left(v \times U^{z}\right) \cdot \overline{U^{z}} d s .
$$

Taking into account the boundary conditions

$$
\begin{gathered}
v \times U^{z}=0 \text { on } \Gamma_{D}, \\
v \times \nabla \times U^{z}=i \frac{\gamma_{b}^{2}}{k_{b}} \lambda\left(v \times U^{z}\right) \times v+\beta_{b} \gamma_{b}^{2} v \times U^{z} \text { on } \Gamma_{I},
\end{gathered}
$$

we have that

$$
I=2 i \frac{\gamma_{b}^{2} \lambda}{k_{b}} \int_{\Gamma_{I}}\left|v \times U^{z}\right|^{2} d s .
$$

Furthermore, in view of the bilinearity of the integral $I$, we have

$$
I=I_{1}+I_{2}+I_{3}+I_{4},
$$


where

$$
\begin{aligned}
I_{1}= & \int_{\partial D}\left[\left(v \times E^{z}\right) \cdot \nabla \times \overline{E^{z}}-\left(v \times \overline{E^{z}}\right) \cdot \nabla \times E^{z}\right] d s \\
& -2 \beta_{b} \gamma_{b}^{2} \int_{\partial D}\left(v \times E^{z}\right) \cdot \overline{E^{z}} d s \\
I_{2}= & -\int_{\partial D}\left[\left(v \times E^{z}\right) \cdot \nabla \times \overline{E_{z}\left(\cdot, q, \gamma_{b}\right)}-\left(v \times \overline{E_{z}\left(\cdot, q, \gamma_{b}\right)}\right) \cdot \nabla \times E^{z}\right] d s \\
& +2 \beta_{b} \gamma_{b}^{2} \int_{\partial D}\left(v \times E^{z}\right) \cdot \overline{E_{z}\left(\cdot, q, \gamma_{b}\right)} d s \\
I_{3}= & -\int_{\partial D}\left[\left(v \times E_{z}\left(\cdot, q, \gamma_{b}\right)\right) \cdot \nabla \times \overline{E^{z}}-\left(v \times \overline{E^{z}}\right) \cdot \nabla \times E_{z}\left(\cdot, q, \gamma_{b}\right)\right] d s \\
& +2 \beta_{b} \gamma_{b}^{2} \int_{\partial D}\left(v \times E_{z}\left(\cdot, q, \gamma_{b}\right)\right) \cdot \overline{E^{z}} d s \\
I_{4}= & \int_{\partial D}\left[\left(v \times E_{z}\left(\cdot, q, \gamma_{b}\right)\right) \cdot \nabla \times \overline{E_{z}\left(\cdot, q, \gamma_{b}\right)}-\left(v \times \overline{E_{z}\left(\cdot, q, \gamma_{b}\right)}\right) \cdot \nabla \times E_{z}\left(\cdot, q, \gamma_{b}\right)\right] d s \\
& -2 \beta_{b} \gamma_{b}^{2} \int_{\partial D}\left(v \times E_{z}\left(\cdot, q, \gamma_{b}\right)\right) \cdot \overline{E_{z}\left(\cdot, q, \gamma_{b}\right)} d s
\end{aligned}
$$

For the evaluation of $I_{1}$, we apply the second vector Green's theorem for the first integral and Gauss' theorem for the second integral for the functions $E^{z}$ and $\overline{E^{z}}$ in $D$ and taking into account that $E^{z}, \overline{E^{z}}$ are solutions of (15) we get $I_{1}=0$. A similar application in $\Omega \backslash \bar{D}$ for $E_{z}\left(\cdot, q, \gamma_{b}\right)$ and $\overline{E_{z}\left(\cdot, q, \gamma_{b}\right)}$ gives

$$
\begin{aligned}
I_{4}= & \int_{\partial \Omega}\left[\left(v \times E_{z}\left(\cdot, q, \gamma_{b}\right)\right) \cdot \nabla \times \overline{E_{z}\left(\cdot, q, \gamma_{b}\right)}-\left(v \times \overline{E_{z}\left(\cdot, q, \gamma_{b}\right)}\right) \cdot \nabla \times E_{z}\left(\cdot, q, \gamma_{b}\right)\right] d s \\
& -2 \beta_{b} \gamma_{b}^{2} \int_{\partial \Omega}\left(v \times E_{z}\left(\cdot, q, \gamma_{b}\right)\right) \cdot \overline{E_{z}\left(\cdot, q, \gamma_{b}\right)} d s .
\end{aligned}
$$

Finally, using the representation (10) with $E_{z}=q \cdot \widetilde{B}(\cdot, z)$ we have that $I_{2}=-q \cdot E^{z}$ and $I_{3}=q \cdot \overline{E^{z}}$. Substituting the values of the integrals $I_{1}$ to $I_{4}$ in (39), we obtain (38).

\section{Conclusions}

In this paper, the reciprocity gap functional method has been employed to reconstruct scatterers with mixed boundary conditions, embedded in a piecewise chiral medium. The importance of this method lies in the fact that we avoid the need to compute Green's function of the background medium. In the basic integral Equation (26) of the method, we have used the chiral Herglotz wave functions, which form a dense set of solutions of (3). The solution $g$ of this equation has been employed to determine the surface impedance. If the chirality measures $\beta_{0}$ and $\beta_{1}$ become zero, then the chirality reciprocity gap functional coincides with the corresponding functional in achiral media. In the future, the present method should be extended to solve inverse transmission problems in chiral media.

Funding: This research received no external funding.

Conflicts of Interest: The author declares no conflict of interest.

Dedication: This paper is dedicated to the inspired mathematician Constantin M. Petridi, who has devoted his life to Mathematics.

\section{References}

1. Ammari, H.; Nédélec, J.C. Time-harmonic electromagnetic fields in thin chiral surved layeres. SIAM J. Math. Anal. 1998, 29, 395-423. [CrossRef]

2. Athanasiadis, C.; Kardasi, E. Beltrami Herglotz functions for electromagnetic scattering theory in chiral media. Appl. Anal. 2005, 84, 145-163. [CrossRef]

3. Athanasiadis, C.; Costakis, G.; Stratis, I.G. Electromagnetic scattering by a homogeneous chiral obstacle in a chiral environment. IMA J. Appl. Math. 2000, 64, 245-258. [CrossRef] 
4. Athanasiadis, C.E.; Sevroglou, V.I.; Skourogiannis, K.I. The inverse electromagnetic scattering problem by a mixed impedance screen in chiral media. Inverse Probl. Imaging 2015, 9, 951-970. [CrossRef]

5. Lakhtakia, A.; Varadan, V.K.; Varadan, V.V. Time-harmonic electromagnetic fields in chiral media. In Lecture Notes in Physics; No 335; Springer: Berlin, Germany,1989.

6. Lakhtakia, A. Beltrami Fields in Chiral Media; World Scientific: Singapore, 1994.

7. Arens, T.; Hagemann, F.; Hettlich, F.; Kirsch, A. The definition and measurement of electromagnetic chirality. Math. Methods Appl. Sci. 2018, 41, 559-572. [CrossRef]

8. Wang, Z.; Cheng, F.; Winsor, T.; Liu, Y. Optical Chiral Metamaterials: a Review of the Fundamentals, Fabrication Methods and Applications. Nanotechnology 2016, 27, 20. [CrossRef]

9. Athanasiadis, C.E.; Sevroglou, V.I.; Skourogiannis, K.I. The direct electromagnetic scattering problem by a mixed impedance screen in chiral media. Appl. Anal. 2012, 91, 2083-2093. [CrossRef]

10. Cakoni, F.; Colton, D. Qualitative Methods in Inverse Elctromagnetic Scattering Theory; Springer: Berlin, Germany, 2005.

11. Athanasiadis, C.; Athanasiadou, E.; Kikeri, E. The reciprocity gap operator for electromagnetic scattering in chiral media. Submitted.

12. Cakoni, F.; Colton, D.; Monk, P. The Linear Sampling Method in Inverse Electromagnetic Scattering; SIAM Society for Industrial and Applied Mathematics: Philadelphia, PA, USA, 2011.

13. Colton, D.; Haddar, H. An application of the reciprocity gap functional to inverse scattering theory. Inverse Probl. 2005, 21, 383-398. [CrossRef]

14. Cakoni, F.; Fares, M.; Haddar, H. Analysis of two linear sampling methods applied to electromagnetic imaging of buried objects. Inverse Probl. 2006, 22, 845-867. [CrossRef]

15. Athanasiadis, C.E.; Natroshvili, D.; Sevroglou, V.; Stratis, I.G. An application of the reciprocity gap functional to inverse mixed impedance problems in elasticity. Inverse Probl. 2010, 26, 085011. [CrossRef]

16. Cakoni, F.; Colton, D. Target identification of buried coated objects. Comput. Appl. Math. 2006, 25, $269-288$.

17. Cakoni, F.; Haddar, H. Identification of partially coated anisotropic buried objects using electromagnetic Cauchy data. J. Integral Equ. Appl. 2007, 19, 359-389. [CrossRef]

18. Sun, Y.; Guo, Y.; Ma, F. The reciprocity gap functional method for the inverse scattering problem for cavities. Appl. Anal. 2016, 95, 1327-1346. [CrossRef]

19. Zeng, F.; Liu, X.; Sun, J.; Xu, L. Reciprocity gap method for an interior inverse scattering problem. J. Inverse Ill-Posed Probl. 2017, 25, 57-68. [CrossRef]

20. Takida, Y.; Koyama, S.; Ueno, N.; Saruwatari, H. Reciprocity gap functional in spherical harmonic domain for gridless sound field decomposition. Signal Process. 2020, 169, 107383 . [CrossRef]

21. Charnley, M.; Wood, A. Object identification in Radar imaging via the reciprocity gap method. Radio Sci. 2020, 55, e2019RS006946. [CrossRef]

22. Di Cristo, M.; Milan, G. Reconstruction of inclusions in electrical conductors. IMA J. Appl. Math. 2020, 85, 933-950. [CrossRef]

23. Faucher, F.; Alessandrini, G.; Barucq, H.; de Hoop, M.V.; Gaburro, R.; Sincich, E. Full reciprocity-gap waveform inversion enabling sparse-source acquisition. Geophysics 2020, 85, R461-R476. [CrossRef]

24. Ghanmi, A.; Mdimagh, R.; Saad, I.B. Identification of points sources via time fractional diffusion equation. Filomat 2018, 32, 6189-6201. [CrossRef]

25. Colton, D.; Kress, R. Inverse Acoustic and Electromagnetic Scattering Theory; Springer: New York, NY, USA, 1998.

26. Dassios, G.; Kleinman, R. Low Frequency Scattering; Oxford University Press, Clarenton Press: Oxford, UK, 2000.

27. Lindell, I.V.; Sihvola, A.H.; Tretyakov, S.A.; Viitanen, A.J. Electromagnetic Waves in Chiral and Bi-isotropic Media; Artech House: Boston, MA, USA, 1994.

28. Monk, F. Finite Element Methods for Maxwell's Equations; Oxford University Press: Oxford, UK, 2003.

29. Cakoni, F.; Colton, D.; Monk, P. The electromagnetic inverse scattering problem for partially coated Lipschitz domains. Proc. R. Soc. Edinb. 2004, 134, 845-867. [CrossRef] 